\title{
Potential rapid assessment of breast cancer prognosis using induced chromosome condensation performed on cytological specimens
}

\author{
Romain Molist ${ }^{1}$, Michelle Gerbault-Seureau ${ }^{1}$, Jerzy Klijanienko ${ }^{2}$, Philippe Vielh ${ }^{2,3}$ and \\ Bernard Dutrillaux ${ }^{1}$ \\ ${ }^{1}$ Cytogénétique Moléculaire et Oncologie, UMR 147, CNRS, Institut Curie, France and ${ }^{2}$ Département de \\ Biologie des Tumeurs, Institut Curie, Paris, France
}

\begin{abstract}
We have previously shown that assessment of chromosome alteration rate by cytogenetics is well correlated with breast cancer prognosis factors. As karyotypes are usually difficult to obtain from solid tumors using conventional methods, a new approach is proposed. Metaphase-like chromosomes were directly obtained following chromosome condensation using calyculin A (okadaic acid) from cytologic specimens of breast cancers sampled by fine needle. Chromosome counts and rearrangement rates were established in a series of 45 tumors, as early as $24-48 \mathrm{~h}$ after sampling. A high rate of rearranged chromosomes was found to correlate with high histological grade, TNM stage and S-phase fraction, loss of estrogen receptor expression and DNA aneuploidy. The indication of genome alteration provided by this method constitutes a simple, potent and early potential prognostic factor in breast cancer directly applied on cytological specimens.
\end{abstract}

Laboratory Investigation (2004) 84, 433-439, advance online publication, 16 February 2004; doi:10.1038/labinvest.3700062

Keywords: breast cancer; cytogenetics; cytology

Extensive data are available on chromosome alterations in breast cancer, obtained after either karyotyping or comparative genomic hybridization (CGH). Recurrent chromosome imbalances have been detected by both methods ${ }^{1-5}$ but the lack of specific alterations, such as those observed in some types of leukemias and sarcomas, has limited the value of cytogenetics for diagnostic purposes.

After short-term culturing and karyotyping, we showed that an increased chromosome rearrangement rate per karyotype correlated with commonly used adverse prognostic factors such as high histologic grade, ${ }^{6}$ loss of steroid hormone receptor expression $^{7}$ and high S-phase fraction. ${ }^{8}$ However, both the complexity of the procedure and the limited rate (about 50\%) of successful cultures limited the practical value of this method for

Correspondence: Dr B Dutrillaux, UMR 147, CNRS, Institut Curie, 26, rue d'Ulm, Paris, Cedex 05, 75248, France.

E-mail : bernard.dutrillaux@curie.fr

${ }^{3}$ Current address: Cytopathologie, Institut Gustave Roussy, France

Received 21 December 2002; revised 24 October 2003; accepted 29 October 2003; published online 16 February 2004 clinical purposes. Furthermore, cultures were developed from surgical biopsies, that is, often after treatment decisions had already been made.

Fine-needle sampling is an early routine procedure for breast cancer diagnosis. ${ }^{9}$ It provides a number of cells sufficient for flow cytometry and hormonal receptor analyses, but insufficient for cell culturing and karyotyping with a reasonable success rate. CGH and other in situ hybridization methods have been applied to this material to determine chromosome imbalances. ${ }^{10,11}$ This provides an interesting tool to detect the presence of malignant cells, but the information remains too limited for diagnostic and prognostic purposes.

Premature chromosome condensation (PCC) can be used to induce metaphase-like chromosomes and improve cytogenetic studies, but the technique remained complex and poorly efficient until it was found that okadaic acid, an inhibitor of protein phosphatases, could induce PCC in various cell types. ${ }^{12}$ This compound is active in all phases of the cell cycle in cells displaying phosphatase activity, inducing G1-, S- or G2-phase PCC. G1-phase PCC leads to monochromatidic chromosomes, which can be identified by chromosome painting. ${ }^{13}$ S-phase 
PCC leads to chaplet-like chromosomes, unsuitable for analysis. G2-phase PCC leads to metaphase-like chromosomes, at various compaction stages. The expected number of 'metaphases' should, therefore, depend on the duration of the G2 phase. Using BrdU incorporation, we showed that cancer cells, particularly breast cancer cells, have a very long (12$17 \mathrm{~h}) \mathrm{G} 2$ phase, as compared to nontransformed cells in culture. ${ }^{14}$ This might represent an advantage for obtaining metaphase-like chromosomes. Okadaic acid-induced PCC was applied to an unselected series of fine-needle samplings performed for breast cancer diagnosis. A reasonable success rate was obtained, providing us with good information on chromosome alterations in cells spontaneously proliferating immediately after sampling. The results obtained are briefly described and discussed in relation to clinical data and classical prognostic factors.

\section{Materials and methods}

Patients were referred to the Institut Curie Cytology Unit for suspicion of breast cancer. After informed consent, the content of one fine-needle sampling from 68 patients was used for cytogenetic analysis, in parallel with routine cytologic examination and flow cytometric analysis of DNA content and cell proliferation. ${ }^{9}$ When available, data on steroid hormone receptor expression, histologic grading and TNM staging were also compared to cytogenetic data.

For cytogenetic analysis, tumor cell suspensions were immediately placed in $5 \mathrm{ml}$ of L15 (Leibovitz) medium (Gibco, Paisly, UK) containing $50 \mu \mathrm{l}$ of heparin solution $(5000 \mathrm{IU} / \mathrm{ml}$, Choay, Gentilly, France). L15 medium (1 ml) containing $0.25 \mu \mathrm{g}$ of calyculin A (okadaic acid, Sigma, Aldrich, France) was added as soon as possible, generally less than $1 \mathrm{~h}$ after sampling. After $40-50 \mathrm{~min}$ at $37^{\circ} \mathrm{C}$, the cell suspension was centrifuged and treated to obtain 'metaphase' spreads according to our usual procedure.$^{15}$ Chromosomes were studied after R-banding. To establish the representative number of chromosomes per tumor, we considered the modal number, or the mean number in the few cases without a modal number. Normal and abnormal chromosomes were counted to determine the percentage of rearranged chromosomes per karyotype. Chromosome painting using whole chromosome 1, 4 (Cambio, Cambridge, UK), 5 and 6 (Oncor, Gaithersburg, USA) probes was applied to three cases to check the feasibility of 'in situ' hybridization on this material. Finally, the presence or absence of homogeneously staining regions (hsr) was recorded.

Both the presence or absence of hsr and the percentage of abnormal chromosomes were studied in relation to clinicopathologic data. In view of the limited number of cases, each parameter compared was distributed into two subsamples as follows: for data with a continuous distribution, such as the rates of rearranged chromosomes and proliferative indexes, a cut-off value was chosen to obtain two subsamples of roughly similar size: $20 \%$ of rearranged chromosomes and an S-phase fraction of $4 \%$. For histologic grading, ${ }^{16}$ we grouped grades I and II, which were compared to grade III tumors. For TNM staging, ${ }^{17}$ we grouped stages I and IIA (low stages), which were compared to stages IIB and III (high stages). For estrogen receptor status, we considered positive estrogen receptor $(\mathrm{ER}+)$ and negative (ER-) tumors, according to the EORTC Receptor Study Group recommendations. ${ }^{7}$ All subsamples were compared two by two using the $\chi^{2}$ test.

Finally, the total number of chromosomes was compared with DNA ploidy obtained by flow cytometry.

\section{Results}

Clinicopathologic and cytogenetic data are summarized in Table 1. Cytogenetic information was obtained in 45 tumors, and other data were available in $36-45$ of these tumors.

\section{Cytogenetics}

Metaphases or pseudometaphases with R-banding suitable for analysis were obtained in $66 \%$ of the cases (45/68). The number of metaphases with a quality of banding sufficient for analysis varied from case to case, but was often limited (less than 10). The 23 unsuccessful assays were due to small number of cells in the samples (seven cases); absence or very small number of metaphases detected; and poor quality of smears and chromosome banding. The quality of chromosome banding was considered to be sufficient for hsr detection in 43 cases and hsr were found in 26 of these cases $(60 \%)$, a percentage similar to that found after cell culture. ${ }^{18}$ The rate of rearranged chromosomes ranged from $0 \%$ to $61 \%$, and the total number of chromosomes ranged from 39 to 120 . A bimodal number of chromosomes was detected in 11 tumors. Overall, the same abnormal chromosomes tended to be observed in the two populations of these tumors, indicating that they were composed of sub-clones developed from a monoclonal tumor. However, in two of these tumors, one 'clone' was composed of cells with a normal karyotype.

Among apparently monoclonal tumors, only two had normal karyotypes. If these tumors are really representative, this would indicate that the rate of breast tumors with a normal karyotype is at most $5 \%(2 / 45)$.

\section{Chromosome Number and DNA Ploidy}

Among 14 diploid, pseudodiploid or near-diploid tumors studied by cytogenetics, 13 were also 
Table 1 Clinicopathologic data of the 45 cases with cytogenetic information

\begin{tabular}{|c|c|c|c|c|c|c|c|c|c|c|c|c|}
\hline Case no. & Age & Meno-pause & Histologic type & Grade & $T N M$ & Stage & $E R$ & S-phase (\%) & DNA indices & Nb. Chr. & Rea \% & $h s r$ \\
\hline 1 & 66 & + & IDC & I & T2No & IIA & ND & 2.5 & 1 & 53 & 0 & - \\
\hline 2 & 67 & + & IDC & II & $\mathrm{NE}$ & $\mathrm{NE}$ & + & 2.8 & 1.16 & 43 & 14 & - \\
\hline 3 & 81 & + & IDC & III & T2No & IIA & - & 4 & 1.59 & 46 & 9 & - \\
\hline 4 & 37 & - & IDC & III & T2No & IIA & - & 25 & 1.65 & $45 / 76$ & 49 & + \\
\hline 5 & 67 & + & IDC & II & T4N1b & IIIB & + & 10 & 1.87 & 70 & 59 & + \\
\hline 6 & 94 & + & IDC & II & T2N1b & IIB & + & 5.8 & 1.95 & $47 / 96$ & 11 & - \\
\hline 7 & 70 & + & IDC & II & T2No & IIA & + & 0.8 & 1 & 46 & 2 & - \\
\hline 8 & 67 & + & IDC & III & T4N1b & IIIB & - & 21 & 1.69 & 66 & 38 & + \\
\hline 9 & 68 & + & IDC & I & T4N1a & IIIB & + & 2.7 & 1.97 & 83 & 6 & - \\
\hline 10 & 70 & + & IDC & I & T2N1a & IIA & + & 15.3 & $1.61 / 2.4$ & 47/Poly & 6 & - \\
\hline 11 & 39 & - & IDC & II & T2No & IIA & + & 2.7 & 1 & 41 & 20 & + \\
\hline 12 & 57 & + & IDC & II & T2N1b & IIB & + & ND & 1.95 & $42 / 84$ & 38 & + \\
\hline 13 & 80 & + & IDC & II & T2No & IIA & + & 7.4 & 1.51 & 64 & 9 & + \\
\hline 14 & 44 & - & IDC & II & T3N1b & IIIB & + & 4.4 & 1 & 41 & 30 & + \\
\hline 15 & 57 & + & IDC & I & T2No & IIA & - & 6.3 & 1.69 & 73 & 22 & + \\
\hline 16 & 37 & - & IDC & II & T2N1a & IIA & + & 4.7 & $1.82 / 1.95$ & 86 & 17 & - \\
\hline 17 & 80 & + & IDC & III & T4No & IIIB & + & 6.5 & 1.54 & $46 / 62$ & 25 & + \\
\hline 18 & 98 & + & IDC & I & T4No & IIIB & + & ND & ND & 84 & 12 & - \\
\hline 19 & 80 & + & IDC & II & T2No & IIA & + & 1.3 & 1 & 44 & 33 & - \\
\hline 20 & 61 & + & IDC & III & T2N1b & IIB & - & 10.7 & 1.87 & $39 / 76$ & 26 & + \\
\hline 21 & 54 & + & IDC & ND & T2N1 & IIB & ND & 14.3 & $1.6 / 3.1$ & $61 / 120$ & 30 & + \\
\hline 22 & 56 & + & IDC & III & T2N1b & IIB & + & 10 & 1.86 & 83 & $>20$ & ND \\
\hline 23 & 40 & - & IDC & ND & T2N1b & IIB & - & 17.5 & 1.51 & 62 & 61 & + \\
\hline 24 & 43 & - & IDC & ND & T4N1a & IIIB & ND & 2.3 & 1.93 & 84 & 21 & + \\
\hline 25 & 39 & - & IDC & III & T3N1b & IIIA & - & ND & $1.9 / 2.77$ & $65 / 120$ & 28 & - \\
\hline 26 & 50 & - & IDC & II & T4N1b & IIIB & ND & 0.6 & 1 & 46 & 2 & - \\
\hline 27 & 90 & + & IDC & II & T2No & IIA & + & 0.8 & 1 & 46 & 0 & - \\
\hline 28 & 46 & - & IDC & I & T2No & IIA & + & 0.5 & 1 & 55 & 36 & + \\
\hline 29 & 79 & + & IDC & III & T3No & IIB & - & 8.3 & 1.6 & $46 / 63$ & $>25$ & + \\
\hline 30 & 38 & - & IDC & III & T2No & IIA & - & ND & 1.4 & 46 & 15 & - \\
\hline 31 & 49 & - & IDC & III & T1N1 & IIA & ND & ND & ND & 44 & 25 & + \\
\hline 32 & 39 & - & IDC & II & T2No & IIA & + & 8.8 & 1 & 47 & 4 & - \\
\hline 33 & 83 & + & IDC & I & T2No & IIA & + & 5.1 & 1 & 78 & 22 & + \\
\hline 34 & 43 & - & IDC & II & T1No & I & ND & 6 & 1.69 & 68 & $>24$ & + \\
\hline 35 & 37 & - & IDC & III & T2N1 & IIB & ND & 19.2 & 1.6 & $46 / 70$ & $>29$ & + \\
\hline 36 & 41 & - & ILC & II & T2N1b & IIB & + & 1.3 & 1 & 40 & 0 & - \\
\hline 37 & 66 & + & ILC & II & T2No & IIA & + & 4.3 & 1.7 & 71 & 14 & + \\
\hline 38 & 55 & + & APO & ND & T3N1b & IIIA & + & 21.6 & 1.75 & 69 & 25 & + \\
\hline 39 & 74 & + & APO & III & T4N3MX & IIIB & - & ND & 1.93 & 46 & 23 & ND \\
\hline 40 & 76 & + & IDC & II & T1N0 & I & ND & ND & ND & $47 / 74$ & 18 & + \\
\hline 41 & & & ISC & High & T1No & I & ND & 3.6 & 1.8 & 69 & 25 & + \\
\hline 42 & 36 & - & IDC & III & T2No & IIA & - & 4.8 & 1.65 & 59 & 33 & + \\
\hline 43 & 71 & + & IDC & II & $\mathrm{T} 2 \mathrm{~N} 1$ & IIB & + & 0.8 & 1 & 46 & 2 & - \\
\hline 44 & 82 & + & IDC & III & T3N1 & IIIA & - & 10.7 & $1.56 / 1.88$ & 56 & 44 & + \\
\hline 45 & 46 & - & IDC & I & T2No & IIA & + & ND & 1.61 & 61 & 31 & + \\
\hline
\end{tabular}

Age at diagnosis (years), menopausal status (post: +); histologic type (WHO classification) IDC: invasive ductal carcinoma; ILC: invasive lobular carcinoma; APO: invasive apocrine carcinoma; ISC: in situ carcinoma; histologic grade; TNM classification and staging, ER: presence (+) or absence (-) of ER expression; S-phase fraction (\%); DNA indices (1:diploid); Nb. Chr.: number of chromosomes; Rea \%: rate of rearranged chromosomes; hsr: presence (+) or absence (-) of homogeneously staining regions. ND: not determined, Poly: polyploid

studied by flow cytometry, 11 of which were found to be DNA diploid. Among 18 hyperploid ( $>50$ chromosomes) tumors studied by cytogenetics, 17 were also studied by flow cytometry, 15 of which were found to be DNA hyperploid. Thus, chromosome counts and DNA ploidy were well, but not perfectly correlated in unimodal tumors.

This correlation was much poorer for bimodal tumors. Among 11 tumors found to be bimodal by cytogenetics, 10 were studied by flow cytometry of which only three were considered to be bimodal. In all discordant cases, the diploid or near-diploid population seen by cytogenetics was not considered to be abnormal by flow cytometry. Only two tumors were found to be bimodal by flow cytometry, but not by cytogenetic analysis. In one case, the two populations had very similar DNA ploidies (DNA indices of 1.82 and 1.95), while a modal chromosome number of 86 was found by cytogenetics.

\section{Chromosome Rearrangements and S-phase Data}

On average, tumors with a high S-phase fraction (greater than $4 \%$ ) provided more metaphases than those with a low S-phase fraction. However, 
metaphases were obtained in five cases with less than $1 \%$ of cells in the $S$ phase. Tumors with high or low S-phase fractions (18 vs 19 tumors) were analyzed in relation to their high or low rate of rearranged chromosomes (21 vs 16 tumors). Tumors with a high S-phase fraction appeared to present more frequently highly rearranged karyotypes than tumors with a low S-phase fraction $\left(\chi^{2}=7.85, \delta=1\right.$, $P<0.005)$.

\section{Chromosome Rearrangements and Histologic Grading}

Among the 40 tumors studied by pathologists, 26 were classified as grade I or II and 14 as grade III. In all, 21 tumors had a high and 19 had a low rate of rearranged chromosomes. Grade III tumors presented a higher rate of rearranged chromosomes than lower grade tumors $\left(\chi^{2}=8.06, \delta=1, P<0.005\right)$. However, four grade I tumors had a high rate of rearranged chromosomes, indicating that gross genomic alterations may occur more rapidly than phenotypic changes.

\section{Chromosome Rearrangements and TNM Staging}

TNM staging of 45 tumors showed 27 high-stage (IIB and III) and 18 low-stage (I and IIA) tumors. Of these 45 tumors, 27 had a high and 18 had a low rate of rearranged chromosomes. High-stage tumors more frequently presented a high rate of rearranged chromosomes than low-stage tumors $\left(\chi^{2}=8.53\right.$, $\delta=1, P<0.005)$.

\section{Chromosome Rearrangements and Estrogen Receptor Status}

The ER status was studied in 36 tumors, 12 of which were considered to have lost their ER expression
(ER-). In this series, 20 had a high and 16 had a low rate of rearranged chromosomes. ER- tumors more frequently possessed high rates of rearranged chromosomes than ER + tumors $\left(\chi^{2}=5.61, \delta=1\right.$, $P<0.02$ ).

Finally, the following parameters indicated in Table 1 were compared two by two: pre- vs postmenopause at diagnosis; low vs high histologic grade, low vs high TNM stage; ER + vs ER-; low vs high S-phase fraction; DNA diploidy vs DNA aneuploidy (flow cytometry); diploid and neardiploid vs hyperploid number of chromosomes; low vs high rate of rearranged chromosomes and presence vs absence of hsr (Figure 1). The menopausal status was not found to be correlated with any other parameter. A high rate of rearranged chromosome was found to be the factor most significantly associated with all other indicators of adverse prognosis, followed by histologic grade III and DNA aneuploidy.

\section{Comparison of Clinicopathological Data from Cases with or without Successful Cytogenetic Analysis}

To know whether tumors successfully studied by this approach were representative or not of the whole series of samples, we compared their clinicopathological data. Rates of high TNM stage, loss of ER, high S-phase fraction, DNA aneuploidy, and of histological grades I, II and III were thus compared between the 45 successful and 21/23 unsuccessful cases (Table 2). These rates are quite similar for DNA aneuploidy, high S-fraction, loss of ER and histological grading I. There are slightly less high TNM stages, and histological grade III and more grade II in unsuccessful than those in successful cases. These differences do not reach statistical significance. This suggests that there is no strong

\begin{tabular}{|c|c|c|c|c|c|c|c|c|}
\hline \multirow{9}{*}{ Menopause } & $\begin{array}{l}\text { Histologic } \\
\text { grade III }\end{array}$ & $\begin{array}{l}\text { High } \\
\text { TNM } \\
\text { stage }\end{array}$ & $\begin{array}{l}\text { Loss of } \\
\text { Estrogen } \\
\text { receptor }\end{array}$ & $\begin{array}{l}\text { High } \\
\text { S-phase } \\
\text { fraction }\end{array}$ & $\begin{array}{c}\text { DNA } \\
\text { aneuploidy }\end{array}$ & $\begin{array}{c}\text { High } \\
\text { Number of } \\
\text { chromosomes }\end{array}$ & $\begin{array}{c}\begin{array}{c}\text { High rate of } \\
\text { rearranged } \\
\text { chromosomes }\end{array} \\
\end{array}$ & $\begin{array}{c}\text { Presence } \\
\text { of } \\
\text { hsr }\end{array}$ \\
\hline & - & - & - & - & - & - & - & - \\
\hline & $\begin{array}{c}\text { Histologic } \\
\text { grade III }\end{array}$ & + & ++ & + & ++ & - & ++ & - \\
\hline & & $\begin{array}{l}\text { High } \\
\text { TNM } \\
\text { stage }\end{array}$ & - & - & - & - & ++ & - \\
\hline & & & $\begin{array}{l}\text { Loss of } \\
\text { Estrogen } \\
\text { receptor }\end{array}$ & - & + & - & + & - \\
\hline & & & & $\begin{array}{l}\text { High } \\
\text { S-phase } \\
\text { fraction }\end{array}$ & ++ & ++ & ++ & + \\
\hline & & & & & $\begin{array}{c}\text { DNA } \\
\text { aneuploidy }\end{array}$ & ++ & + & + \\
\hline & & & & & & $\begin{array}{c}\text { High number } \\
\text { of } \\
\text { chromosomes }\end{array}$ & + & + \\
\hline & & & & & & & $\begin{array}{c}\text { High rate of } \\
\text { rearranged } \\
\text { chromosomes }\end{array}$ & ++ \\
\hline
\end{tabular}

Figure 1 Relationships between the various criteria indicated in Table 1. For instance, a high rate of rearranged chromosomes was not significantly correlated with menopausal status at diagnosis $(P>0.05:-)$, but was significantly $(0.05>P \geq 0.01$ : +$)$ correlated with loss of ER expression, DNA aneuploidy and number of chromosomes (above 50), and very significantly $(P<0.01:++)$ correlated with high histologic grade, high TNM stage, high S-phase fraction and presence of hsr. 
Table 2 Comparison of clinicopathological data in tumors with $(n=45)$ or without $(n=21)$ successful cytogenetic study (in percentage)

\begin{tabular}{|c|c|c|c|c|c|c|c|}
\hline & \multicolumn{3}{|c|}{ Histological grading } & \multirow{2}{*}{ High TNM stage } & \multirow{2}{*}{ Loss of ER } & \multirow{2}{*}{ High S-phase fraction } & \multirow{2}{*}{ DNA aneuploidy } \\
\hline & $I$ & $I I$ & III & & & & \\
\hline Successful & 21 & 47 & 32 & 50 & 33 & 65 & 71 \\
\hline Unsuccessful & 22 & 67 & 11 & 40 & 35 & 60 & 70 \\
\hline
\end{tabular}

bias of analysis, and that our data as fairly representative of breast cancer status at diagnosis.

\section{Chromosome Painting at Various Stages of Compaction}

To check the possibility of applying chromosome painting to artificially condensed chromosomes from fresh, uncultured tumors, we used chromosome 1, 4, 5 and 6 probes on cases 20, 30 and 37 . As expected, this method allowed detection of aberrations such as translocations of chromosomes 1 and 5 (case no. 20, Figure 2a) and chromosomes 1 and 6 (case no. 37, Figure 2b). Various stages of compaction of normal chromosomes 4 and 6 (case No. 30) are also shown: S-phase PCC with heterogeneous compaction in Figure 2c, and G2/M-phase compaction in Figure 2d.

\section{Discussion}

Despite the limited amounts of cytological material provided by fine-needle sampling, we obtained analyzable metaphase-like chromosomes in about two-thirds of the cases, using chemically induced chromosome condensation. As fine-needle sampling fails to provide sufficient tumor material for any investigation in about $10 \%$ of cases, this represents a fairly good success rate, which can probably be improved by better adaptation of the chemical treatment. The comparison of clinicopathological data between successfully or unsuccessfully studied tumors suggests the absence of strong bias of analysis. In particular, the rates of histological grade I, or low S-phase fractions were very similar, which indicates that the low proliferative capacities of the tumors does not constitute a strong limitation for the method used here. Chromosome banding and painting could be applied, without any particular difficulty, within the $24 \mathrm{~h}$ following collection of tumor material. This method, therefore, provides a good tool to study rapidly breast cancer genome alterations. It can probably be applied to other tumor types in which fine- needle samplings are routinely performed for diagnosis.

In this limited series of tumors, karyotypes were established on cycling cells at the time of fineneedle sampling, that is, without any artifact related to 'in vitro' cell selection. The results obtained are
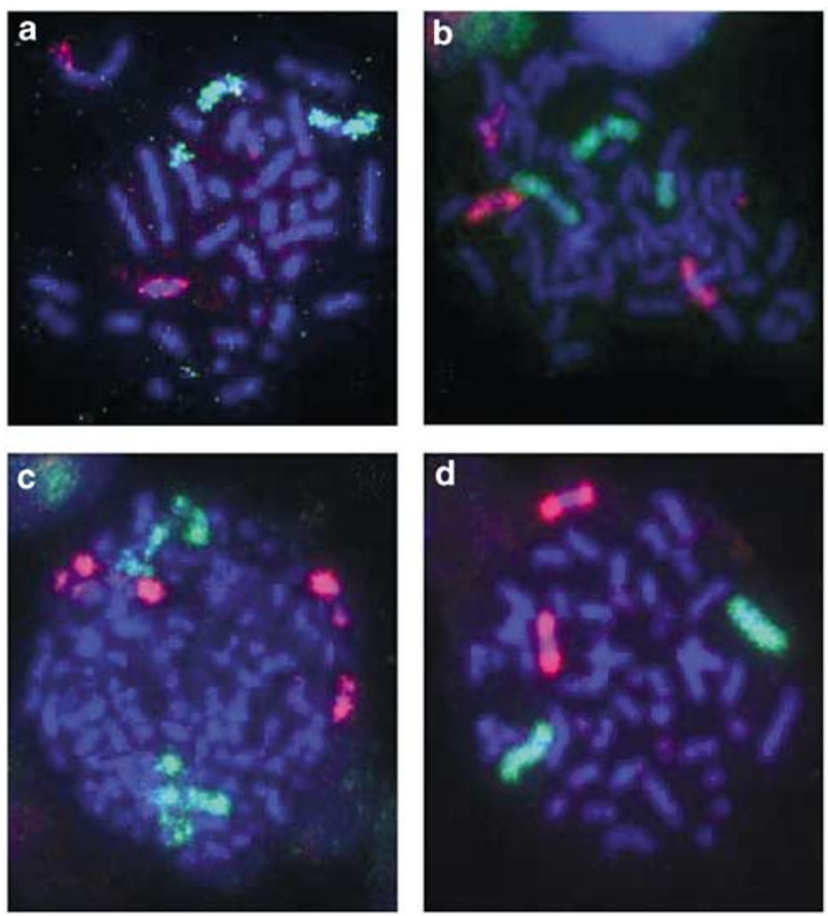

Figure 2 Paintings of (a) chromosomes 1 (green) and 5 (red) (case no. 20), (b) chromosomes 1 (green) and 6 (red) (case no. 37), (c) and (d) chromosomes 4 (green) and 6 (red) (case no. 30).

fairly similar to those obtained after short-term (1-3 days) culture, ${ }^{1}$ but the proportion of tumors with abnormal karyotypes is higher. All cases with abnormal karyotypes exhibited chromosome imbalances, whereas in most published series, the rate of tumors with nonclonal, generally balanced alterations, or no chromosome imbalance is at least $20 \% .{ }^{19}$ The data obtained after chemically induced PCC may therefore provide the best 'in vivo' representation of the tumor karyotype. It would indicate that only a small minority of breast cancers present either normal or only balanced karyotypes.

Several years ago, we proposed that karyotypic data established after short-term cell culture of surgical biopsies could represent a good prognostic criterion. A high rate of rearranged chromosomes was found to correlate with high cell proliferation, ${ }^{8}$ loss of steroid hormone receptor expression, ${ }^{7}$ high histologic grade, ${ }^{6}$ and high epidermal growth factor receptor (EGFR) expression (unpublished data).

Tumors with a low rate of rearranged chromosomes would therefore have a better prognosis than 
those with a high rate of rearranged chromosomes. These parameters, although all correlated with the rate of rearranged chromosomes, were found to be either weakly or not correlated with each other. The same conclusions apply to this series of tumors. The relationships between the rate of rearranged chromosomes and parameters such as the histologic grading, the S-phase fraction and ER expression, which are statistically significant despite a limited number of cases studied, may indicate a better detection of chromosome alterations after PCC than following cell culture.

The practical value of chromosome analysis has been severely limited to date by the low success rate (about $50 \%$ ), the complexity of the procedure and the need to obtain a surgical specimen. The results were therefore often obtained after treatment decisions had already been made. After chemically induced PCC, karyotypic data can be established within $24 \mathrm{~h}$ following fine-needle sampling, which is often the first diagnostic procedure. The quality of the chromosomes is, however, frequently low, and this method does not aim to replace other cytogenetic or molecular studies, which may provide more accurate information.

However, the quantification of chromosome alterations may have some interests as compared to other prognostic factors. It is a nonspecific, thus independent parameter, which is a consequence of a progressive accumulation of chromosome rearrangements. Its high rate either indicates a fast or a long tumor progression or both. This notion of tumor evolution is not found in other parameters. Its lack of specificity is also an advantage as compared to parameters depending on whole or nothing events. For instance, loss of ER expression is generally correlated with adverse prognosis, but it is not an obligatory step. Thus other pejorative events may have occurred before it, explaining some lack of correlation. Finally, the quantification of chromosome alterations may be performed as soon as $24 \mathrm{~h}$ after the fine-needle sampling and provides malignancy diagnosis and prognosis indications. This may substantially improve therapeutic decisions. This could be particularly interesting in breast cancer without palpable lymph node. For instance, in this series of patients in which 21 had no palpable lymph nodes, 11 tumors had a highly rearranged karyotype. If our conclusions are valid, this may indicate their adverse prognosis, and give useful information for their treatment decision.

\section{Acknowledgement}

The present study was supported in part by Institut Curie 'PIC - cancer du sein'. We thank all pathologists of the Institut Curie for grading and estrogen receptor expression determination of the tumors.

\section{References}

1 Dutrillaux B, Gerbault-Seureau M, Zafrani B. Characterization of chromosomal anomalies in human breast cancer. A comparison of 30 paradiploid cases with few chromosome changes. Cancer Genet Cytogenet 1990; 49:203-217.

2 Kallioniemi A, Kallioniemi OP, Sudar D, et al. Comparative genomic hybridization for molecular cytogenetic analysis of solid tumors. Science 1992;258: 818-821.

3 Pandis N, Jin Y, Gorunova L, et al. Chromosome analysis of 97 primary breast carcinomas: identification of eight karyotypic subgroups. Genes Chromosomes Cancer 1995;12:173-185.

4 Tirkkonen M, Tanner M, Karhu R, et al. Molecular cytogenetics of primary breast cancer by CGH. Genes Chromosomes Cancer 1998;21:177-184.

5 Teixeira MR, Pandis N, Heim S. Cytogenetic clues to breast carcinogenesis. Genes Chromosomes Cancer 2002;33:1-16.

6 Dutrillaux B, Gerbault-Seureau M, Remvikos Y, et al. Breast cancer genetic evolution: I. Data from cytogenetics and DNA content. Breast Cancer Res Treat 1991;19:245-255.

7 Magdelenat H, Gerbault-Seureau M, Laine-Bidron C, et al. Genetic evolution of breast cancer: II. Relationship with estrogen and progesterone receptor expression. Breast Cancer Res Treat 1992;22:119-127.

8 Remvikos Y, Gerbault-Seureau M, Magdelenat H, et al. Proliferative activity of breast cancers increases in the course of genetic evolution as defined by cytogenetic analysis. Breast Cancer Res Treat 1992;23:43-49.

9 Vielh P, Magdelenat H, Mosseri V, et al. Immunocytochemical determination of estrogen and progesterone receptors on 50 fine-needle samples of breast cancer. A prospective study including biochemical correlation and DNA flow cytometric analysis. Am J Clin Pathol 1992;97:254-261.

10 Truong K, Guilly MN, Gerbault-Seureau M, et al. Quantitative FISH by image cytometry for the detection of chromosome 1 imbalances in breast cancer: a novel approach analyzing chromosome rearrangements within interphase nuclei. Lab Invest 1998;78: 1607-1613.

11 Heselmeyer-Haddad K, Chaudhri N, Stoltzfus P, et al. Detection of chromosomal aneuploidies and gene copy number changes in fine needle aspirates is a specific, sensitive, and objective genetic test for the diagnosis of breast cancer. Cancer Res 2002;62: 2365-2369.

12 Yamashita K, Yasuda H, Pines J, et al. Okadaic acid, a potent inhibitor of type 1 and type $2 \mathrm{~A}$ protein phosphatases, activates cdc2/H1 kinase and transiently induces a premature mitosis-like state in BHK21 cells. EMBO J 1990;9:4331-4338.

13 Durante M, Furusawa Y, Gotoh E. A simple method for simultaneous interphase-metaphase chromosome analysis in biodosimetry. Int J Radiat Biol 1998;74: 457-462.

14 Dutrillaux B, Gerbault-Seureau M, Zafrani B, et al. Prolonged G2 phase of breast cancer cells and chromosome damage. Eur J Cancer 1991;27:1307-1312.

15 Dutrillaux BCJ, (eds). La Pratique de L'analyse Chromosomique. Masson: Paris, 1981.

16 Elston CW, Ellis IO. Pathological prognostic factors in breast cancer. I. The value of histological grade in 
breast cancer: experience from a large study with longterm follow-up. Histopathology 1991;19:403-410.

17 UICC. TNM, Classification of Malignant Tumours, 5th edn. Wiley-Liss: New York, 1997.

18 Bernardino J, Apiou F, Gerbault-Seureau M, et al. Characterization of recurrent homogeneously staining regions in 72 breast carcinomas. Genes Chromosomes Cancer 1998;23:100-108.

19 Kleivi K, Lothe RA, Heim S, et al. Genome profiling of breast cancer cells selected against in vitro shows copy number changes. Genes Chromosomes Cancer 2002;33: 304-309. 